\title{
Glycemic Variability in Type 1 Diabetes Compared with Degludec and Glargine on the Morning Injection: An Open-label Randomized Controlled Trial
}

\author{
Ryo Iga - Hiroshi Uchino (D) - Ken Kanazawa - Shuki Usui · \\ Masahiko Miyagi · Naoki Kumashiro - Hiroshi Yoshino • \\ Yasuyo Ando · Takahisa Hirose
}

Received: December 10, 2016/ Published online: May 25, 2017

(C) The Author(s) 2017. This article is an open access publication

\section{ABSTRACT}

Introduction: Optimal adjustment of basal insulin to overcome hypoglycemia and glycemic variability (GV) depends on its duration of action and peak-less profile. Owing to the ability of long-acting basal insulin to avoid hypoglycemia, we titrated pre-meal glucose to normal fasting blood glucose, $80-110 \mathrm{mg} / \mathrm{dL}$ $(4.5-6.1 \mathrm{mmol} / \mathrm{L})$, and post-meal glucose to $80-140 \mathrm{mg} / \mathrm{dL}(4.5-7.8 \mathrm{mmol} / \mathrm{L})$. The purpose of this study was to evaluate two basal insulin analogues degludec (IDeg) and glargine (IGlar), injected in the morning, for GV using continuous glucose monitoring (CGM) in type 1 diabetes (T1DM).

Methods: In this crossover study, 20 Japanese patients with T1DM (age $54 \pm 16$ years, disease duration $16 \pm 8$ years, BMI $24 \pm 4 \mathrm{~kg} / \mathrm{m}^{2}$,

Enhanced content To view enhanced content for this article go to http://www.medengine.com/Redeem/ 6B48F0607D2935C5.

Electronic supplementary material The online version of this article (doi:10.1007/s13300-017-0269-0) contains supplementary material, which is available to authorized users.

R. Iga · H. Uchino $(\bowtie) \cdot$ K. Kanazawa · S. Usui · M. Miyagi - N. Kumashiro - H. Yoshino - Y. Ando · T. Hirose

Division of Diabetes, Metabolism and

Endocrinology, Department of Internal Medicine,

Toho University School of Medicine, Tokyo, Japan e-mail: h.uchino@med.toho-u.ac.jp
HbA1c $7.4 \pm 0.8 \%)$ were randomized into one of two different starting regimens, and CGM was conducted on three consecutive days during the last week of each 12-week titration period. Treatment satisfaction was assessed at the end of each treatment period using the Diabetes Therapy-Related Quality of Life Questionnaire (DTR-QOL).

Results: There were no differences in HbA1c, total insulin dosage, body weight changes, and basal to bolus ratio between the IDeg and IGlar arms. The day-to-day variability in fasting interstitial GV on the CGM curves was significantly less in the IDeg than IGlar treatment period $\quad(25.9 \pm 22.0$ vs. $43.8 \pm 30.1 \mathrm{mg} / \mathrm{dl}$, $p=0.04)$. Other markers of $\mathrm{GV}$, calculated by the EasyGV software, including mean amplitude of glycemic excursions (MAGE), J-index, total and nocturnal hypoglycemia were not different between the two treatment periods. The score of "satisfaction with treatment", a subdomain of the DTR-QOL system, was higher in the IDeg period.

Conclusion: Thus, the morning injection of the two long-acting insulin analogues seemed similar with regard to the magnitude of hypoglycemia in T1DM, but treatment with IDeg was associated with lower day-to-day variation in glucose level. These results suggest that IDeg is safe with minimal morning GV in patients with T1DM.

Clinical trial registration: Japanese Clinical Trials Registry, UMIN000012358. 
Keywords: Degludec; Glargine; Glycemic variability; Type 1 diabetes

\section{INTRODUCTION}

Clinical evidence indicates that intensive insulin therapy reduces the risk of long-term complications in patients with type 1 diabetes mellitus (T1DM) [1]. However, such therapy is associated with increased risk of hypoglycemia and unawareness of hypoglycemia, in part through the modification of glucose-sensing neurons [2, 3]. Previous studies indicated that low hemoglobin A1c (HbA1c) and short-term variations in blood glucose are independent risk factors for hypoglycemia [4, 5]. However, lack of awareness of occasional episodes of hypoglycemia, such as nocturnal hypoglycemia, is not uncommon, especially in the absence of clinical signs and symptoms [6]. Continuous glucose monitoring (CGM) is particularly useful to identify episodes of hypoglycemia and variability in glycemic level including episodes of both hypo- and hyperglycemia [7]. The normal ranges of glycemic variability (GV) recoded by CGM have been published for normal subjects [8] and patients with T1DM [9].

Insulin analogue degludec (IDeg) is a formulated insulin analogue of di-hexamers that is reorganized into multi-hexamer compounds at the site of subcutaneous tissue injection [10], and later degraded to monomers at a slow rate followed by absorption into the systemic circulation [10]. On the basis of our clinical experience, patients with T1DM tend to avoid the use of a single dose of another insulin analogue glargine (IGlar) before breakfast with multiple bolus injections, one for each meal, because of fear of possible insufficient action of IGlar until the next morning. To our knowledge, there are no randomized crossover designed clinical trials that have compared glycemic control in T1DM under treatment with long-acting IDeg and IGlar injection "before breakfast".

Hypoglycemia and fear of hypoglycemia are some of the major concerns among patients, limiting their quality of life (QOL) and their ability to achieve glycemic targets [11-13]. The objective of this study was to compare the effects of IDeg and IGlar, in the context of GV measured by CGM, including total time spent in hypoglycemia and mean glycemic level. In addition, we used the Diabetes Therapy-Related Quality of Life Questionnaire to assess the duration of hypoglycemia and GV associated with the two insulin analogues, as well as the extent of satisfaction with each treatment [14].

\section{METHODS}

\section{Study Subjects}

This study was conducted in accordance with the International Conference on Harmonization Guidelines for Good Clinical Practice and the Declaration of Helsinki [15]. The study was approved by Toho University ethics committee. All procedures followed were in accordance with the ethical standards of the responsible committee on human experimentation (institutional and national) and with the Helsinki Declaration of 1964, as revised in 2013. Informed consent was obtained from all patients for being included in the study.

This randomized, open-label, crossover study was conducted to determine the effects of IDeg and IGlar treatment once daily in the morning in lowering fasting blood glucose (FBG) and the severity of potential hypoglycemia (Figs. 1, 2). Patients were randomized to receive either IDeg or IGlar, employing identical adjustment algorithms. In the latter approach, the data from the first arm of the study were combined with those of the second arm, at the end of the study. The protocol for this trial and supporting CONSORT checklist are available as supporting information; see Checklist S1 and Protocol S1 (original language) and S2 (English translation). Recruitment took place between 2013 and 2014 and patients were followed up until December 2014. On the basis of a pilot CGM conducted in 2013 by the Toho University, Tokyo, a minimum difference in CGM parameters between the IDeg and IGlar arms over a month was expected. To achieve a power of $80 \%$, and with a significance level of $5 \%(\alpha=0.05)$, a minimum of 10 participants in each arm with a crossover design was required (total $n=20$ to calculate). Eligible 


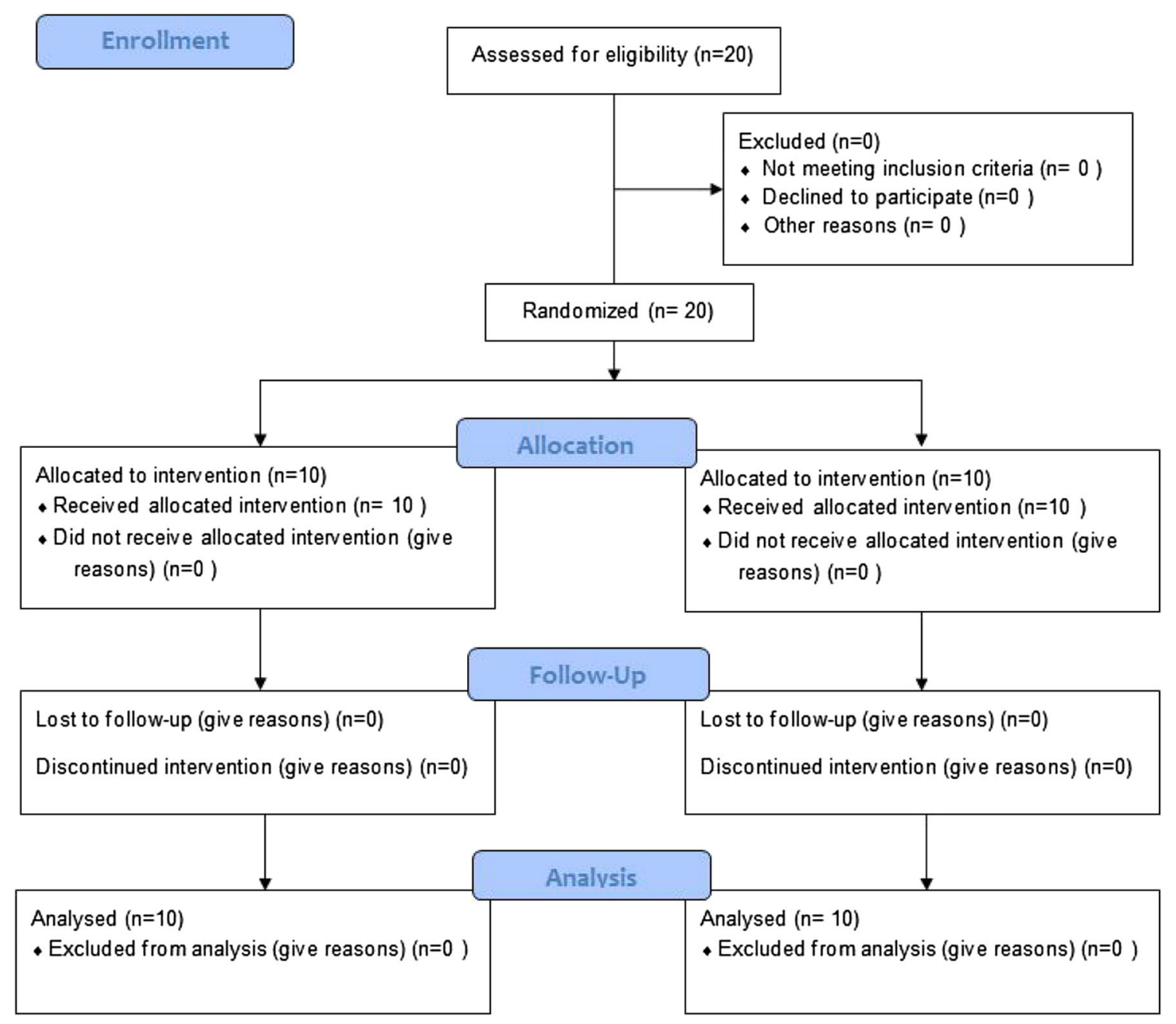

Fig. 1 Enrollment of participants in the study

patients were aged 20 years or older with history of T1DM for at least 1 year. The exclusion criteria included pregnant women, history or presence of active cancer or cardiovascular disease, stroke, blood pressure beyond the normal range (systolic greater than $180 \mathrm{mmHg}$; diastolic greater than $100 \mathrm{mmHg}$ ), proliferative retinopathy or macroangiopathy or active infectious disease such as hepatitis B and C infections.

Patients were also excluded if they had ever been diagnosed with type 2 diabetes, had hemoglobin less than $11 \mathrm{~g} / \mathrm{dL}$, platelet count less than $10 \times 10^{9} / \mathrm{L}$, fasting triglycerides greater than $500 \mathrm{mg} / \mathrm{dL}$ (greater than $5.7 \mathrm{mmol} / \mathrm{L}$ ), liver disease, alanine aminotransferase (ALT), or aspartate aminotransferase (AST) level of more than 2.5-fold the upper limit of normal, renal transplantation or serum creatinine greater than $2.0 \mathrm{mg} / \mathrm{dL}$ (greater than $177 \mathrm{mmol} / \mathrm{L}$ ), more than two episode of severe hypoglycemia within the preceding 12 months or diagnosis of hypoglycemia unawareness, participation in a weight loss program, or at least two emergency room visits or hospitalization for poor glucose control within the preceding 6 months.

At baseline (before study entry), all 20 patients were treated during the prestudy period (12 weeks) with insulin aspart (IAsp) and basal insulin. IAsp was later continued at a dose of insulin titration algorithms, as stated below, throughout the 24-week duration of the study (Fig. 2). The study included 20 participants who were randomized by computer-generated 


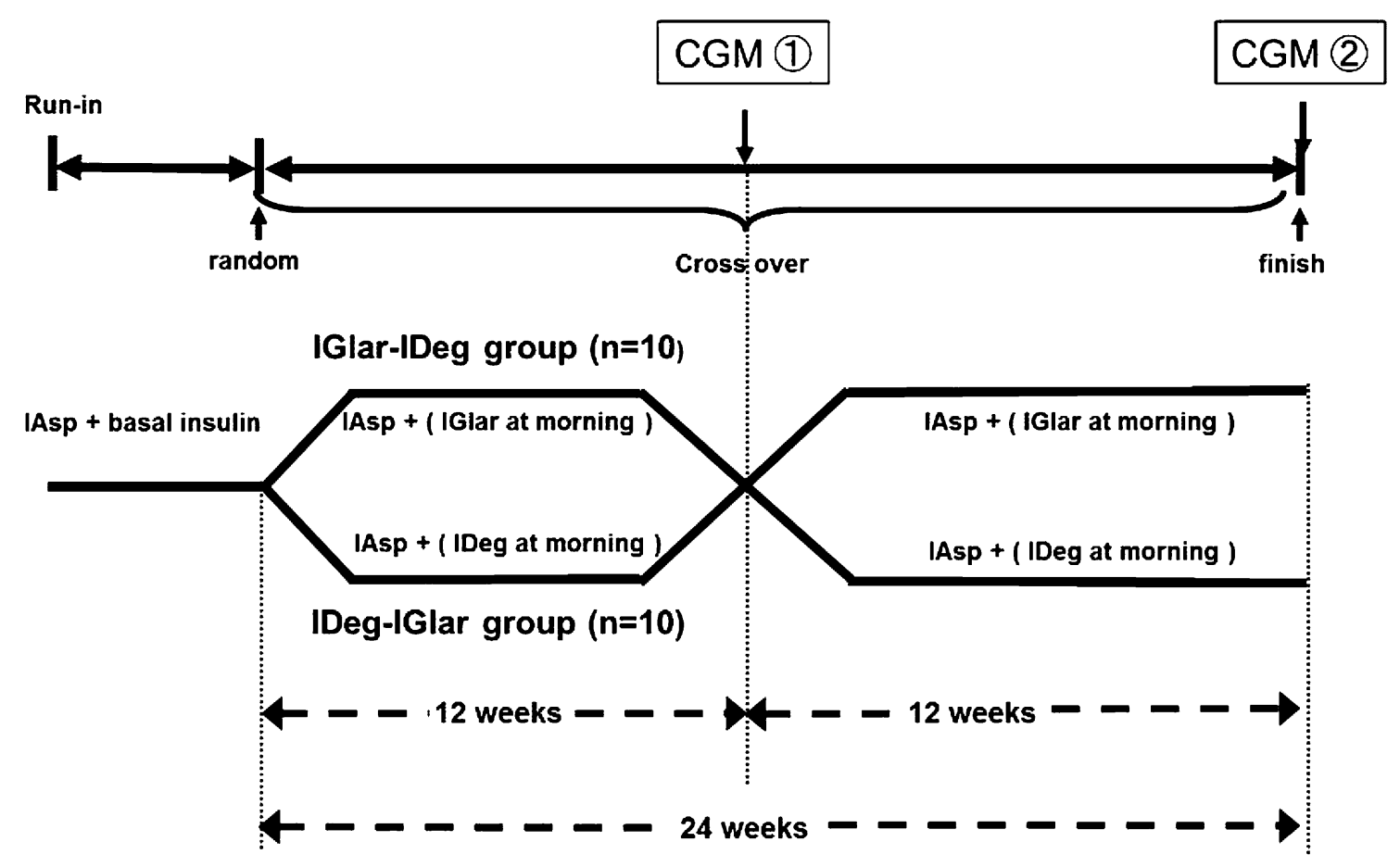

Fig. 2 Study design. IAsp insulin aspart, IDeg insulin degludec, IGlar insulin glargine

assignment to receive first either IDeg $(n=10)$ or IGla $(n=10)$ continuously for 12 weeks. CGM was performed in a blinded fashion on three consecutive days $(72-84 \mathrm{~h})$ of the last week of the 12-week study period (Fig. 1). CGM is able to record glucose values for 5 days; however, to complete 24 -h recording sets, we used 3 days in the middle. Then basal insulin was switched from IDeg to IGla and vice versa from IGla to IDeg and treatment was continued for another 12 weeks. Again, CGM was performed in the same way as the first arm of the study. A Medtronic Diabetes (Northridge, CA) CGMS Gold continuous glucose recorder was used.

\section{Outcome Measures and Measurements}

To evaluate the incidence of hypoglycemia during treatment with IDeg and IGlar, we measured GV using CGM in T1DM patients. Hypoglycemia was defined as CGM of less than $70 \mathrm{mg} / \mathrm{dL}$ (less than $3.9 \mathrm{mmol} / \mathrm{L}$ ). The time spent in hypoglycemia at interstitial glucose (IG) value of less than $70 \mathrm{mg} / \mathrm{dL}$ (less than
$3.9 \mathrm{mmol} / \mathrm{L}$ ) per 24 -h period was expressed as total hypoglycemia time (\%). The nocturnal period for the CGM assessment represented the time between 2400 and 0600 hours, and the time spent at IG value of less than $70 \mathrm{mg} / \mathrm{dL}$ was described as nocturnal hypoglycemia (\%). The percentage of time spent with IG value between 80 and $140 \mathrm{mg} / \mathrm{dL} \quad(4.4-7.8 \mathrm{mmol} / \mathrm{L})$ was expressed as percentage of time spent at optimum glucose (\%). Between- and within-day glucose SD during the nocturnal period and the daytime period (0600-2400 hours) was calculated to assess the within-patient GV. The term "between-day variability" as used here and previously [16] corresponds to the previously designated term of "standard deviation between daily means" [17]. The mean amplitude of glycemic excursions (MAGE) was calculated from the CGM taking into account the glycemic peaks and nadirs recorded during the $24 \mathrm{~h}$, beyond the average glucose values [18]. The J-index was calculated using the glycemic average and SD and used to describe GV [19]. On the basis of the time required to achieve steadystate circulating IDeg and previous studies on 
IGlar [20], all basal insulin were restricted not to increase the dose before the time-to-onset, which need the 3-day interval.

The target fasting and postprandial blood glucose levels used to optimize the dose of insulin were $80-110 \mathrm{mg} / \mathrm{dL} \quad(4.5-6.1 \mathrm{mmol} / \mathrm{L})$ and $80-140 \mathrm{mg} / \mathrm{dL} \quad(4.5-7.8 \mathrm{mmol} / \mathrm{L})$, respectively. The insulin algorithm, adapted from previous studies $[16,20]$, was based on the mean fasting blood glucose of three consecutive mornings before the visit: for a mean FBG of $110-130 \mathrm{mg} / \mathrm{dL}(6.1-7.2 \mathrm{mmol} / \mathrm{L}), 131-160 \mathrm{mg} /$ $\mathrm{dL} \quad(7.3-8.9 \mathrm{mmol} / \mathrm{L})$, and for greater than $161 \mathrm{mg} / \mathrm{dL}$ (greater than $9.0 \mathrm{mmol} / \mathrm{L}$ ), the IDeg and IGlar doses were increased by 2,3 , and 4 IU, respectively. Bolus insulin titration was adopted over three consecutive days: for mean 2-h postprandial blood glucose value of 140-180 mg/dL (7.8-10 mmol/L), 181-220 mg/ dL (10.1-12.2 $\mathrm{mmol} / \mathrm{L})$, and for greater than $220 \mathrm{mg} / \mathrm{dL}$ (greater than $12.2 \mathrm{mmol} / \mathrm{L}$ ), the bolus insulin dose was increased by 2,3 , and $4 \mathrm{IU}$, respectively.

The dose was reduced by $2 \mathrm{IU}$ in patients taking either IDeg or IGlar who experienced at least two or more hypoglycemic episodes with blood glucose values of less than $80 \mathrm{mg} / \mathrm{dL}$ (less than $4.4 \mathrm{mmol} / \mathrm{L}$ ).

Treatment satisfaction was assessed at the end of each treatment period using the Diabetes Therapy-Related Quality of Life Questionnaire (DTR-QOL) [14].

\section{Statistical Analysis}

All analyses were performed using the SAS (SAS Institute, Cary, NC, USA) with the intent-totreat principle based on all patients who were randomized and took at least one dose of the study drug. All tests performed were two-sided at a prespecified a level of 0.1 , and the corresponding $90 \%$ CIs were calculated. No adjustments for multiplicity were performed. The mean duration (in minutes) of individual hypoglycemic episodes according to treatment type and duration (12 weeks) was summarized and compared between the treatment periods using the Student $t$ test. A $P$ value less than 0.05 denoted the presence of a significant difference.

\section{RESULTS}

\section{Subject Characteristics}

The clinical characteristics of the two groups are presented in Table 1. Age, duration of diabetes (years), body mass index (BMI), HbA1c (\%), total daily dose of insulin (TDD) (IU/kg/day), and basal insulin percentage of TDD were similar in the two groups.

\section{Glycemic Control}

As shown in Table 2, the average HbA1c (\%), body weight (kg), BMI, TDD, and basal insulin percentage of TDD were not significantly different at each 12-week endpoint of the treatment periods. Within each 12 -week study period, the mean total daily dose of insulin (IU/ kg/day) and percentage of basal insulin to the TDD (\%) were similar in both treatment periods. Table 3 shows the 24-h CGM data expressed as the mean glucose value $(\mathrm{mg} / \mathrm{dL})$, which was identical in both treatment periods $(p=0.435)$. The time spent at optimum glucose (\%) representing the IG between 70 and $140 \mathrm{mg} / \mathrm{dL}(3.9-7.8 \mathrm{mmol} / \mathrm{L})$ was similar in the two treatment periods $(p=0.434)$. The percentage of time in hypoglycemia per $24 \mathrm{~h}$ and nocturnal hypoglycemia (\%) were not different between the two treatment periods $(p=0.300, p=0.325)$. MAGE and J-Index were also identical in the two periods $(p=0.355, p=0.444)$. Table 3 also shows fasting IG and day-to-day variation in fasting IG. While the mean fasting IG was similar in the two insulin treatment periods $(p=0.102)$, the magnitude of day-to-day variation in fasting IG variability was significantly higher in the IGlar treatment period than the IDeg period $(p=0.037)$. Figure 3 provides a schematic representation of the CGM findings.

\section{Diabetes Quality of Life}

We measured the total and four-subdomain scores of the original DTR-QOL (Fig. 4). While the scores of the total and subdomain of DTR-QOL (for item "burden on social activities and daily activities", "anxiety and 
Table 1 Characteristics of patients of the IDeg-IGlar and IGlar-IDeg groups at baseline

\begin{tabular}{lllll}
\hline & Total & IDeg-IGlar & IGlar-IDeg & $\boldsymbol{p}$ value \\
\hline$n$ (male/female) & $20(11 / 9)$ & $10(5 / 5)$ & $10(6 / 4)$ & \\
Age (years) & $54 \pm 16$ & $55 \pm 14$ & $53 \pm 18$ & 0.383 \\
Diabetes (years) & $15.2 \pm 8.5$ & $14.4 \pm 8.6$ & $16.1 \pm 8.7$ & 0.333 \\
Body weight $(\mathrm{kg})$ & $62.0 \pm 11.1$ & $64.0 \pm 10.4$ & $60.0 \pm 12.0$ & 0.218 \\
BMI $\left(\mathrm{kg} / \mathrm{m}^{2}\right)$ & $23.7 \pm 4.3$ & $24.4 \pm 4.4$ & $23.1 \pm 4.1$ & 0.256 \\
HbA1c $(\%)$ & $7.4 \pm 0.8$ & $7.1 \pm 0.9$ & $7.7 \pm 0.6$ & 0.077 \\
Total daily dose of insulin (U/kg) & $0.68 \pm 0.27$ & $0.64 \pm 0.23$ & $0.72 \pm 0.32$ & 0.239 \\
Basal insulin percentage of TDD (\%) & $41.4 \pm 18.2$ & $47.4 \pm 21.2$ & $34.9 \pm 16.1$ & 0.689 \\
\hline
\end{tabular}

Data are mean $\pm \mathrm{SD}$

$T D D$ mean total daily dose in $\mathrm{IU} / \mathrm{kg} / \mathrm{day}$

Table 2 Measures of mean body weight, body mass index, HbAlc, and dose of insulin at 12 weeks post-treatment with IDeg and IGlar periods

\begin{tabular}{lllll}
\hline & Total & IDeg & IGlar & $\boldsymbol{p}$ value \\
\hline$n$ & 40 & 20 & 20 & \\
Body weight $(\mathrm{kg})$ & $61.7 \pm 12.7$ & $61.5 \pm 12.5$ & $61.9 \pm 12.9$ & 0.945 \\
BMI $\left(\mathrm{kg} / \mathrm{m}^{2}\right)$ & $23.6 \pm 4.6$ & $23.5 \pm 4.7$ & $23.6 \pm 4.6$ & 0.884 \\
HbAlc $(\%)$ & $7.2 \pm 0.9$ & $7.2 \pm 0.8$ & $7.3 \pm 0.9$ & 0.733 \\
Total daily dose of insulin (U/kg) & $0.64 \pm 0.2$ & $0.62 \pm 0.21$ & $0.65 \pm 0.2$ & 0.916 \\
Basal insulin percentage of TDD (\%) & $42.3 \pm 16.3$ & $42.5 \pm 16.9$ & $42.1 \pm 15.7$ & 0.755 \\
\hline
\end{tabular}

Data are mean \pm SD of the same insulin treatment group at 12 weeks post-treatment

dissatisfaction with treatment", and "hypoglycemia") were not different between the treatments, the scores of "satisfaction with treatment" subdomain improved significantly during treatment with IDeg $(p<0.009)$.

\section{DISCUSSION}

The main purpose of the present study was to evaluate the effects of morning injection of two long-acting insulin analogues, IDeg and IGlar, on the severity of hypoglycemia and GV using CGM in patients with T1DM. A recent study using CGM suggested that patients with successfully treated T1DM (i.e., HbA1c less than
$7.0 \%)$ spent more than $90 \mathrm{~min} /$ day at blood glucose level below $70 \mathrm{mg} / \mathrm{dL}$ [21]. Although no severe hypoglycemia was observed in the present study, the percentage of time spent in hypoglycemia per $24 \mathrm{~h}$ was similar under both IDeg and IGlar. Our results, however, showed a higher percentage of time in nocturnal hypoglycemia (between 2400 and 0600 hours; nearly $30 \%$ of the time spent in IG less than $70 \mathrm{mg} / \mathrm{dL}$, less than $3.9 \mathrm{mmol} / \mathrm{L}$ ) under both basal insulin arms, compared with that of a previous study [21]. The different results were probably related to the study design; we applied strict blood glucose titration, allowing with fasting self-monitoring of blood glucose (SMBG) values between 80 and $110 \mathrm{mg} / \mathrm{dL}(4.4-6.1 \mathrm{mmol} / \mathrm{L})$. 
Table 3 Results of CGM analysis at 12 weeks after treatment with IDeg and IGlar

\begin{tabular}{llll}
\hline & IDeg & IGlar & $\boldsymbol{p}$ value \\
\hline Number of CGM records & 20 & 20 & \\
Mean glucose value (mg/dL) & $140.1 \pm 40.5$ & $143.2 \pm 28.4$ & 0.435 \\
Optimum glucose value, 70-140 mg/dL (\%) & $50.5 \pm 21.2$ & $49.3 \pm 18.8$ & 0.434 \\
Total hypoglycemia (\%) & $6.6 \pm 9.2$ & $5.4 \pm 6.5$ & 0.300 \\
Nocturnal hypoglycemia (\%) & $39.6 \pm 30.6$ & $35.1 \pm 25.3$ & 0.325 \\
Mean amplitude of glycemic excursions (MAGE) & $159.7 \pm 65.6$ & $168.5 \pm 57.7$ & 0.355 \\
J-index & $41.2 \pm 24.5$ & $42.3 \pm 18.1$ & 0.444 \\
Mean fasting interstitial glucose (mg/dL) & $117.7 \pm 44.4$ & $137.1 \pm 30.5$ & 0.102 \\
Day-to-day variation of fasting interstitial glucose (mg/dL) & $25.9 \pm 22.0$ & $43.8 \pm 30.1$ & 0.037 \\
\hline
\end{tabular}

Data are mean \pm SD

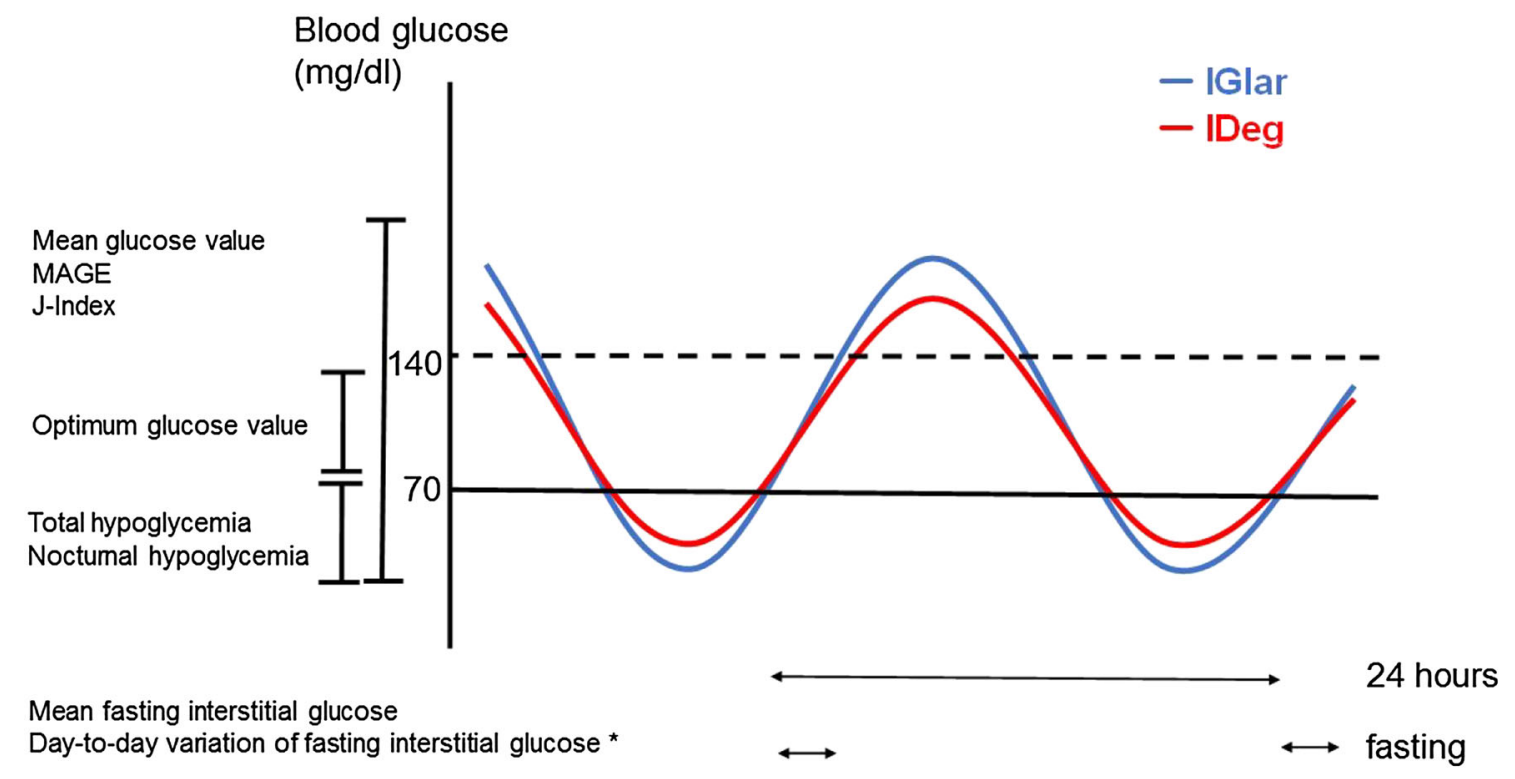

Fig. 3 Schematic representation of the CGM findings. ${ }^{*} p<0.05$

Using similar safety endpoints, including hypoglycemia (which was defined as SMBG values of $101-120 \mathrm{mg} / \mathrm{dL}$ or $5.5-6.7 \mathrm{mmol} / \mathrm{L}$ ), Bergenstal et al. [22] demonstrated previously that LY2605541 (peglispro) improved fasting blood glucose values, with a time spent at hypoglycemia of $11 \%$ only, compared with $38 \%$ under insulin glargine. Thus, the caveats of insulin titration algorithm used in clinical trials could potentially pose serious hypoglycemia risk.

Currently, there is no gold standard for evaluation of GV. Indeed, assessment of GV in routine clinical practice remains a challenge at present. Especially in patients with T1DM, the achievement of tight glycemic control with limited intraday variability in blood glucose level remains a challenge. However, the values 


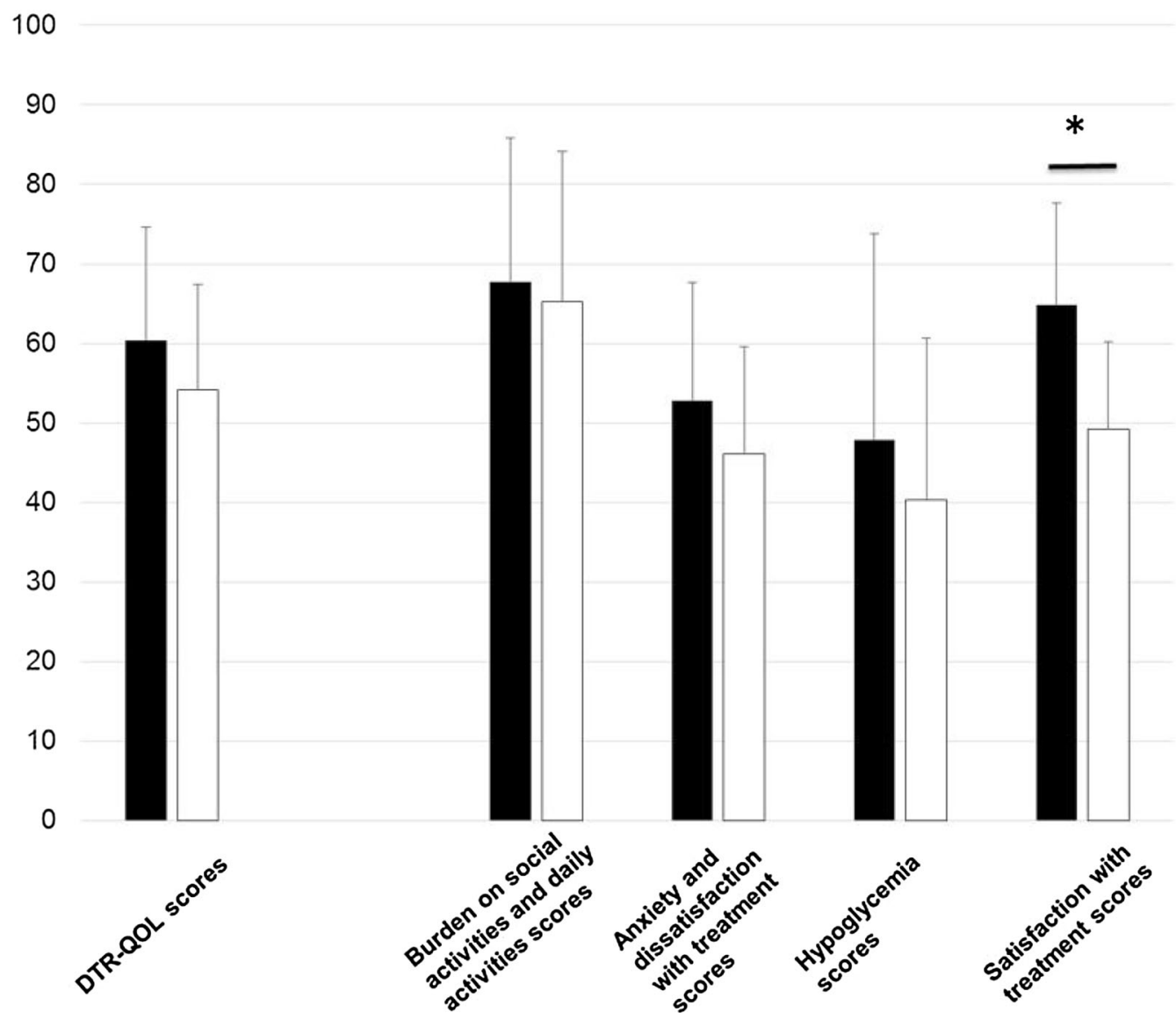

Fig. 4 Comparison of total and subdomain scores of DTR-QOL between IDeg and IGlar groups (20 DTR-QOL records for each treatment period). Data are mean \pm SD. Higher scores indicate better quality of life

of mean glucose level, optimum glucose level, MAGE, J-Index, and fasting interstitial glucose in this study were similar between the two treatment periods. Thus, IDeg and IGlar both tended to improve day-to-day variation in fasting interstitial GV. In this regard, Heise et al. [23] reported that the day-to-day variability was smaller in patients on IDeg compared to those on IGlar under steady-state conditions. The difference could be due to the longer duration of action and lower peak-to-trough ratio of IDeg than IGlar [24]. Further studies are needed to evaluate the therapeutic application of IDeg.

In the present study, we used the Japanese version of the DTR-QOL questionnaire for Japanese patients with T1DM. This version has been validated previously [14]. The total DTR-QOL score was comparable in the two insulin arms. The score for the component "satisfaction with treatment" was significantly higher in the IDeg treatment period. Our results showed that the T1DM treatment-related DTR-QOL questionnaire may help in clinical decision-making regarding the selection of the most appropriate type of insulin for patients with T1DM. Physicians should pay attention to treatment-related QOL especially in patients on insulin therapy because poor GV may result in inadequate satisfaction with the treatment.

Our study has certain limitations. First, our randomized crossover study was open-labeled, which carries concerns regarding drug-specific bias. Second, the percentage time and severity of hypoglycemia were monitored only over a relatively short period of time. Third, to some extent the A1c difference between both groups at baseline was substantial and the results could have been affected by the large SD and small 
number of study participants. The results need to be confirmed in long-term prospective studies of large numbers of patients.

\section{CONCLUSIONS}

Compared to IGla, morning injection of long-acting insulin IDeg seems to be associated with lower risk of day-to-day variation in fasting interstitial GV, as well as higher satisfaction with treatment in patients with T1DM.

\section{ACKNOWLEDGMENTS}

No funding or sponsorship was received for this study or publication of this article. All authors made significant contributions to study design, analysis, and interpretation of data, as well as preparing and reviewing the manuscript. All named authors meet the International Committee of Medical Journal Editors (ICMJE) criteria for authorship for this manuscript, take responsibility for the integrity of the work as a whole, and have given final approval for the version to be published. Previous presentation: some of the findings from this study were presented at the 57th Annual Meeting of The Japan Diabetes Society, Osaka, Japan, May 22-24, 2014.

Disclosures. Takahisa Hirose has received honoraria for scientific lectures from MSD, Eli Lilly, Takeda Pharmaceutical Co, Dainippon Sumitomo Pharma, Sanofi-Aventis, Ono Pharmaceutical Co, AstraZeneca, Novo Nordisk Pharma, and Daiichi Sankyo Inc. and also research funds from Eli Lilly, Novo Nordisk Pharma, Kissei Pharma, Boehringer Ingelheim, AstraZeneca, Tanabe Mitsubishi Pharma, and Sanofi. Ryo Iga, Hiroshi Uchino, Ken Kanazawa, Shuki Usui, Masahiko Miyagi, Naoki Kumashiro, Hiroshi Yoshino, and Yasuyo Ando have nothing to disclose.

Compliance with Ethics Guidelines. All procedures followed were in accordance with the ethical standards of the responsible committee on human experimentation (institutional and national) and with the Helsinki
Declaration of 1964, as revised in 2013. Informed consent was obtained from all patients for being included in the study.

Data Availability. The datasets during the current study are available from the corresponding author on reasonable request.

Open Access. This article is distributed under the terms of the Creative Commons Attribution-NonCommercial 4.0 International License (http://creativecommons.org/licenses/ by-nc/4.0/), which permits any noncommercial use, distribution, and reproduction in any medium, provided you give appropriate credit to the original author(s) and the source, provide a link to the Creative Commons license, and indicate if changes were made.

\section{REFERENCES}

1. The Diabetes Control and Complications Trial Research Group. The effect of intensive treatment of diabetes on the development and progression of long-term complications in insulin-dependent diabetes mellitus. N Engl J Med. 1993;329(14):977-86.

2. Garfield AS, Shah BP, Madara JC, et al. A parabrachial-hypothalamic cholecystokinin neurocircuit controls counter regulatory responses to hypoglycemia. Cell Metab. 2014;20(6):1030-7.

3. Diggs-Andrews KA, Zhang X, Song Z, Daphna-Iken $\mathrm{D}$, Routh $\mathrm{VH}$, Fisher SJ. Brain insulin action regulates hypothalamic glucose sensing and the counter regulatory response to hypoglycemia. Diabetes. 2010;59(9):2271-80.

4. Campbell MD, Walker M, Trenell MI, et al. Lowglycemic index meal and bedtime snack prevents postprandial hyperglycemia and associated rises in inflammatory markers, providing protection from early but not late nocturnal hypoglycemia following evening exercise in type 1 diabetes. Diabetes Care. 2014;37(7):1845-53.

5. Phillip M, Battelino T, Atlas E, et al. Nocturnal glucose control with an artificial pancreas at a diabetes camp. N Engl J Med. 2013;368(9):824-33.

6. Sejling AS, Kjær TW, Pedersen-Bjergaard U, et al. Hypoglycemia-associated changes in the electroencephalogram in patients with type 1 diabetes and normal hypoglycemia awareness or unawareness. Diabetes. 2015;64(5):1760-9. 
7. Kropff J, Del Favero S, Place J, et al. 2 Month evening and night closed-loop glucose control in patients with type 1 diabetes under free-living conditions: a randomised crossover trial. Lancet Diabetes Endocrinol. 2015;3(12):939-47.

8. Chan CL, Pyle L, Newnes L, Nadeau KJ, Zeitler PS, Kelsey MM. Continuous glucose monitoring and its relationship to hemoglobin A1c and oral glucose tolerance testing in obese and prediabetic youth. J Clin Endocrinol Metab. 2015;100(3):902-10.

9. Sherr J, Tamborlane WV, Xing D, et al. Achievement of target A1C levels with negligible hypoglycemia and low glucose variability in youth with short-term type 1 diabetes and residual $\beta$-cell function. Diabetes Care. 2012;35(4):817-20.

10. Jonassen I, Havelund S, Hoeg-Jensen T, Steensgaard DB, Wahlund PO, Ribel U. Design of the novel protraction mechanism of insulin degludec, an ultra-long-acting basal insulin. Pharm Res. 2012;29(8):2104-14.

11. Trento M, Trevisan M, Coppo E, et al. Diagnosis of type 1 diabetes within the first five years of life influences quality of life and risk of severe hypoglycemia in adulthood. Acta Diabetol. 2014;51(3):509-11.

12. Cooke D, O'Hara MC, Beinart $\mathrm{N}$, et al. Linguistic and psychometric validation of the diabetes-specific quality-of-life scale in UK English for adults with type 1 diabetes. Diabetes Care. 2013;36(5):1117-25.

13. Pouwer F, Hermanns N. Insulin therapy and quality of life. A review. Diabetes Metab Res Rev. 2009;25(Suppl 1):S4-10.

14. Mashitani T, Hayashino Y, Okamura S, et al. Diabetes treatment-related quality of life is associated with levels of self-care activities in insulin injection among Japanese patients with type 2 diabetes: diabetes Distress and Care Registry at Tenri (DDCRT 8). Acta Diabetol. 2015;52(4):639-47.

15. World Medical Association Declaration of Helsinki. Ethical principles for medical research involving human subjects. JAMA. 2000;284(23):3043-5.
16. Bergenstal RM, Rosenstock J, Arakaki RF, et al. A randomized, controlled study of once-daily LY2605541, a novel long-acting basal insulin, versus insulin glargine in basal insulin-treated patients with type 2 diabetes. Diabetes Care. 2012;35(11):2140-7.

17. Rodbard D. Interpretation of continuous glucose monitoring data: glycemic variability and quality of glycemic control. Diabetes Technol Ther. 2009;11(Suppl 1):S55-67.

18. Monnier L, Mas E, Ginet C, et al. Activation of oxidative stress by acute glucose fluctuations compared with sustained chronic hyperglycemia in patients with type 2 diabetes. JAMA. 2006;295(14):1681-7.

19. Wójcicki JM. "J"-index. A new proposition of the assessment of current glucose control in diabetic patients. Horm Metab Res. 1995;27(1):41-2.

20. Yki-Järvinen $H$, Kauppinen-Mäkelin R, Tiikkainen $\mathrm{M}$, et al. Insulin glargine or NPH combined with metformin in type 2 diabetes: the LANMET study. Diabetologia. 2006;49(3):442-51.

21. Juvenile Diabetes Research Foundation Continuous Glucose Monitoring Study Group, Beck RW, Hirsch IB, et al. The effect of continuous glucose monitoring in well-controlled type 1 diabetes. Diabetes Care. 2009;32(8):1378-83.

22. Bergenstal RM, Rosenstock J, Bastyr EJ 3rd, Prince MJ, Qu Y, Jacober SJ. Lower glucose variability and hypoglycemia measured by continuous glucose monitoring with novel long-acting insulin LY2605541 versus insulin glargine. Diabetes Care. 2014;37(3):659-65.

23. Heise T, Nosek L, Bøttcher SG, Hastrup H, Haahr H. Ultra-long-acting insulin degludec has a flat and stable glucose-lowering effect in type 2 diabetes. Diabetes Obes Metab. 2012;14(10):944-50.

24. Heise T, Nosek L, Roepstorff C, Chenji S, Klein O, Haahr H. Distinct prandial and basal glucose-lowering effects of insulin degludec/insulin aspart (IDegAsp) at steady state in subjects with type 1 diabetes mellitus. Diabetes Ther. 2014;5(1):255-65. 